\title{
Predicted Dynamic and Structural Criterion of Evaluation of Gravitational Waves
}

\author{
M. Zhussupov \\ Science, Noosphereorder Co., London, UK
}

mjalmata@mail.ru

(Received Feb 2017; Published March 2017)

\begin{abstract}
The cosmic (physical) vacuum according to modern data possesses the properties of a material medium. In combination with the celestial bodies, this medium is capable of forming dynamic structures. Presumably, these structures have a wave nature, and in their development, the gravitational waves, emitted by the stars and their planetary systems can take part. It is believed that physical vacuum is almost weightless matter. However this study postulates its high density, as a condition for adaptation of the fundamental physical laws for different mediums and substances. The product of this density and gravitational constant gives vent to the frequency, which is entitled as the probable frequency of the gravitational wave background of space and equals to $.06 \mathrm{~Hz}$ (rounded). The supposed spectrum of gravity waves of the solar system could become a model for the spectral analysis of extra-solar systems and the evaluation of habitable planets.
\end{abstract}

Keywords: Cosmic Vacuum, Gravitational Waves, Frequency, Density Of Physical Vacuum, Exoplanets

DOI:10.14331/ijfps.2017.3300100

\section{INTRODUCTION}

The cosmic vacuum, which is identical to the physical vacuum, is a continuous host medium for all the objects of the Universefrom the elementary particles to the celestial bodies. Recent experimental studies testify that this medium has physical properties characterizing it as a matter. This nature, for example, was confirmed by the Casimir effect, which is manifested by a power pressure from the side of physical vacuum upon two close parallel-uncharged plates (Wilson et al., 2011). A research project carried out at CERN (at the Large Hadron Collider) showed the ability to quantization of the physical vacuum to form massive bosons (Noyes, 2014). It has been postulated that this physical vacuum possesses an extremely high value of the density, which is three orders of magnitude higher than the density of the heaviest chemical elements (Zhussupov, 2015). Such a high density of a medium provides the compatibility of the laws of celestial mechanics and hydrodynamics. At the same time, because of the structural features of the atoms and molecules, the environment of the physical vacuum remains sufficiently permeable for a substance to move relatively easily. During 2015, in Laser Interferometer Gravitational-wave Observatory (LIGO), two big splashes in gravitational waves were registered (Abbott et al., 2016). Besides these solitary waves, according to modern concepts, there is a constant gravitational wave background in the Universe. Now it is sure that the detection of this hypothetical background is not a long way from now. The main criterion for the search and evaluation of habitable exoplanets is the similarity of their dynamic structures with our solar system. Gravitational waves extending for long distances can be a source of information on these structures. Therefore, in the present paper an attempt has been made to calculate the probability of existence of constant 
radiation of gravitational waves by the solar system. In this attempt, we propose the mathematical modeling of the dynamics and structure of the host medium of the observed objects.

\section{SIMULATION OF DYNAMIC AND STRUCTURE OF GRAVITATIONAL WAVES AT THE SOLAR SYSTEM}

Orbital rotation of planets considering the materiality of the physical vacuum is similar to the movement of a substance in cyclones. This involves centrifugal and centripetal forces; however, spatial situation and behavior of the planets are probably a reflection of the symmetry of the host medium. This symmetry is caused by the structure of the oscillation of gravitational waves. The physical vacuum fills not only the space between the objects, but also between the elementary particles in atoms and molecules. It is important to note that the relative proportions of the size of electrons and radius of atoms are comparable to the distances between the stars and planets. In this perspective, the gravitational waves equally influence to the macroscopic bodies and the elementary particles. As a model of a gravitational wave, the elementary volume of the medium of physical vacuum is taken oscillating along a vector of the acceleration created by this wave. In the simplest case, it is possible to consider this volume on Earth surface in the form of vertically vibrating cube, where the lower face is located parallel to the horizontal plane, and the side edges coincide with the direction of a vector of gravity acceleration. If conditionally this container is filled with dusty material, as is known from mechanics, a foreign body in this substance will experience the acceleration of vibration in a direction to acceleration of gravity. The equation for finding of this body in a suspended condition would be (Eq. 1).

$$
g_{C}=\omega^{2} R \text {, }
$$

where $g_{C}$ is the acceleration of vibration; $\omega$ is the frequency of physical vacuum oscillating; $R$ is the oscillation amplitude

The frequency of supposed gravitational waves follows from equality of the received acceleration with acceleration from Newton's law of universal gravitation (Eq. 2).

$$
\omega^{2} R=G \frac{M}{R^{2}} ; \omega_{\mathrm{C}}=\sqrt{G \frac{M}{R^{3}}},
$$

where $G$ is the gravitational constant, $M$ is mass of the pulling object; $R$ is the oscillation amplitude

The relation standing to the right of a gravitational constant has the dimension of density $(D)$, whose numerical value is equal to $5.313 \mathrm{e}^{7} \mathrm{~kg} / \mathrm{m}^{3}$ (Zhussupov, 2015). The solution of Equation 2 gives the value of the oscillation frequency as $0.0595 \mathrm{~Hz}$. Theoretically, the simulated oscillation are the free oscillations of the environment, i.e., cosmic gravitational wave background or relic gravitational waves. Perhaps, the waves of such character were known earlier, but their nature was interpreted incorrectly. For example, unclear existence of infragravity ocean waves with frequencies lower than the wind waves (about $0.05 \mathrm{~Hz}$ ) (Herbers, Elgar, Guza, \& O'Reilly, 1995). Rosetta's Plasma Consortium has uncovered the oscillations in the magnetic field in the comet's environment with 'being sung' at $0.04-0.05 \mathrm{~Hz}$ (Rosetta blog, 2014) . Symbolically, the Foucault pendulum in the Pantheon, Paris oscillates with the frequency of $.06 \mathrm{~Hz}$, although the period of its oscillations depends on the length of the suspension. The interaction of gravitational waves with celestial bodies occurs basically due to the electrons in the shells of atoms that constitute the bodies, as the size of protons and neutrons is too small for their acceptance in calculation. From mechanics, we know that if the source of the waves in any medium oscillates, then each object within this environment also performs harmonic oscillations with the same frequency as the wave source. Therefore, in principle, any observed objects can serve as an indicator of the gravitational waves of the corresponding frequency. The rest mass of the electron is small, but its probable interaction with the gravitational waves can be shown against the background of massive bodies such as planets. In accordance with the laws of conservation of energy and momentum, the reaction of material objects against rolling of gravitational wave should appear in their acceleration. At the same time with acceleration, the matter itself becomes a source of energy and gravitational radiation. The electron as the object having mass according to the law of universal gravitation is associated with planet by force (Eq. 3)

$$
F=G \frac{m_{E} m_{e}}{r^{2}}
$$

where $m_{E}$ is the mass of Earth, $m_{e}$ is the mass of an electron, $r$ is distance between an electron and the center of Earth.

The power capacity of the gravitational radiation of the earth in a pair with an electron at rest is equal to the product of this force on the orbital speed of the Earth. Given this, the vacuum dielectric permittivity (electric constant), power of gravitational radiation is expressed as (Eq. 4).

$$
P_{1}=G \frac{m_{E} m_{e}}{r^{2}} v \varepsilon_{0}
$$

where $v$ is the orbital speed of the Earth, $\varepsilon_{0}$ is electric constant

The energy of the system emitting gravitational waves in terms of frequency for single electron will equal to $h \omega$, where $h$ is Planck's constant. Since power is the rate of consumption energy and taken as angular velocity, power will directly proportional to the square of the frequency (Eq. 5).

$$
P_{2}=h \omega^{2} \text {, }
$$

From these two magnitudes of power, the frequency can be derived as (Eq. 6).

$$
\omega=\sqrt{G \frac{m_{E} m_{e}}{h r^{2}} v \varepsilon_{0}},
$$

The numerical value of this frequency within the accuracy of the gravitational constant coincides with the frequency of the supposed gravitational wave background. Equation 6 reflects the frequency of a single electron in the gravitational field of a planet. For the objects consisting of a larger quantity of electrons as a part of atoms and molecules, it is necessary to increase the mass of rest of an electron by its quantity that corresponds approximately to the total weight of the substance with respect to the proton mass. Magnitude of mass is included in the numerator and denominator and reduced as (Eq. 7).

$$
\omega=\sqrt{G \frac{m_{\mathrm{C}} m_{p}}{h r^{2}} v \varepsilon_{0}},
$$

where $m_{\mathrm{C}}$ is mass of the pulling object, $m_{p}$ is mass of proton, $r$ is radius of an orbit.

From this equation, it follows that the frequency of the emission of gravitational waves does not depend on the mass of the orbital object, but in an inverse proportion depends on the radius of its orbit. The spectral line of gravitational radiation of the Moon according to this equation, where $v$ will already the orbital speed of Earth, is equal to the frequency of $0.0422 \mathrm{~Hz}$. This value is $\sqrt{2}$ times less than the value of own 
oscillations of the physical vacuum obtained above. The wavelength corresponding to this frequency $(\lambda=c / \omega$, where $\mathrm{c}$ is speed of light) without residue, in the style of standing waves, three times fits into lunar orbit. The gravitational frequencies of all planets of solar system, calculated on the basis of Equation 7 (where the pulling object is the Sun), are presented in Table 1. To solve this equation, the data on the numerical value of the speed of translational motion of the sun are required. In a study (Crutcher, 1982), it has been shown that the solar system, as a structural element of the space, is moving relative to interstellar medium at a speed of $25 \pm 3$ $\mathrm{km} / \mathrm{s}$. The results of further calculations depend on the speed of the sun, which considerably varies in different studies. From Equation 6, the frequency of an electron on the surface of the earth is equal to $0.0595 \mathrm{~Hz}$. If it is assumed that this value reflects the frequency of the Earth's gravitational waves, then the necessary speed of the Sun should be equal to about 27 $\mathrm{km} / \mathrm{s}$. According to this speed, the frequencies of gravitational waves that are associated with planets of solar system are found (Tab. 1). Probably the gravitational waves are involved in the process of the orbital rotation of the planets around the sun. In this case, the work of such wave, by analogy with liquids, will equal to product of the acceleration, mass, and amplitude of gravitational wave (Eq. 8).

$$
W_{W}=a M A \text {, }
$$

where $a$ is acceleration of planets toward to Sun, $M$ is mass of a wave, $A$ is amplitude of wave.

In this equation, for the acceleration of the gravitational wave, the acceleration due to gravity of the planets to the sun is taken, whose value is known. The operating mass of gravitational wave is accepted equal to the mass of the physical vacuum which is forced out by the planet. The volume of displaced physical vacuum is not equal to the actual volume of the planet, due to the uneven distribution of electron density in atoms and molecules. The space between the elementary particles in atoms is free and permeable to physical vacuum. The maximum density of the electrons in atoms theoretically can be achieved if all electrons will moved to the level of radius of ion of the highest valence. These values for ionic radii of some chemical elements begin from 8 pm (Grimes, 1976). Therefore as the effective size for the interaction with gravitational waves, the size of ion is taken rather than atom. Because the molecular composition of the planets is not quantifiable, it is presented in the form of hydrogen-like structural elements in an amount equal to the number of protons in the composition of the planet $\left(m_{E} / m_{p}\right)$. The radius of a single structural element in the calculation is assumed to be $8.9 \mathrm{pm}$, so an effective volume for gravitational interactions of the planet is equal to the total volume of these elements. The relations of observed volume of the planet to the effective volume $\left(V_{P} / V_{E}\right)$ are also given in the table. As can be seen from the table that attitude correlates with the values of the average density of the planets $(\rho)$. The operating mass of gravitational wave $\left(M_{W}\right)$, which is equal to the product of the density of physical vacuum and the effective volume $\left(D V_{E}\right)$, is almost two orders of magnitude greater than the actual mass of the planet $(M p l)$. As a result, the numerical value of the work of gravitational waves $\left(W_{W}\right)$ is obtained, which has been shown in Table 1 equal to the kinetic energy of the orbital motion of the planets $\left(E_{K}=m v^{2} / 2\right)$.

Table 1. Simulation parameters of gravitational waves in the solar system

\begin{tabular}{|l|l|l|l|l|l|l|l|l|}
\hline Planets & Mercury & Venus & Earth & Mars & Jupiter & Saturn & Uranus & Neptune \\
\hline$\omega(\mathrm{Hz})$ & 0.154 & 0.0827 & 0.0595 & 0.0392 & 0.0115 & 0.00625 & 0.00311 & 0.00198 \\
\hline$\lambda(\mathrm{m})$ & $1.947 \mathrm{e}^{9}$ & $3.626 \mathrm{e}^{9}$ & $5.036 \mathrm{e}^{9}$ & $7.654 \mathrm{e}^{9}$ & $2.612 \mathrm{e}^{10}$ & $4.794 \mathrm{e}^{10}$ & $9.642 \mathrm{e}^{10}$ & $1.51 \mathrm{e}^{11}$ \\
\hline$\rho \mathrm{kg} / \mathrm{m}^{3}$ & 5427 & 5243 & 5515 & 3933 & 1326 & 687 & 1270 & 1638 \\
\hline$V_{P} / V_{E}$ & 103.2 & 110.4 & 102.3 & 143.8 & 427.3 & 822.9 & 444.9 & 345.6 \\
\hline$a m / c^{2}$ & 0.004 & 0.0011 & 0.00059 & 0.00025 & $2.2 \mathrm{e}^{-4}$ & $6.5 \mathrm{e}^{-5}$ & $1.6 \mathrm{e}^{-5}$ & $6.6 \mathrm{e}^{-6}$ \\
\hline$M p l(\mathrm{~kg})$ & $3.302 \mathrm{e}^{23}$ & $4.869 \mathrm{e}^{24}$ & $5.974 \mathrm{e}^{24}$ & $6.419 \mathrm{e}^{23}$ & $1.899 \mathrm{e}^{27}$ & $5.685 \mathrm{e}^{26}$ & $8.685 \mathrm{e}^{25}$ & $1.024 \mathrm{e}^{26}$ \\
\hline$M_{W}(\mathrm{~kg})$ & $3.12 \mathrm{e}^{25}$ & $4.601 \mathrm{e}^{26}$ & $5.645 \mathrm{e}^{26}$ & $6.065 \mathrm{e}^{25}$ & $1.794 \mathrm{e}^{29}$ & $5.372 \mathrm{e}^{28}$ & $8.206 \mathrm{e}^{27}$ & $9.679 \mathrm{e}^{27}$ \\
\hline$W_{W}(\mathrm{~J})$ & $3.813 \mathrm{e}^{32}$ & $3.02 \mathrm{e}^{33}$ & $2.668 \mathrm{e}^{33}$ & $1.886 \mathrm{e}^{32}$ & $1.634 \mathrm{e}^{35}$ & $2.667 \mathrm{e}^{34}$ & $2.026 \mathrm{e}^{33}$ & $1.525 \mathrm{e}^{33}$ \\
\hline$E_{K}(\mathrm{~J})$ & $3.828 \mathrm{e}^{32}$ & $2.932 \mathrm{e}^{33}$ & $2.668 \mathrm{e}^{33}$ & $1.874 \mathrm{e}^{32}$ & $1.622 \mathrm{e}^{35}$ & $2.651 \mathrm{e}^{34}$ & $2.014 \mathrm{e}^{33}$ & $1.515 \mathrm{e}^{33}$ \\
\hline
\end{tabular}

Theoretically, each planet in association with the sun is presented by its own frequency of gravitational oscillation, at the same time the Sun possesses all ranges of the oscillations noted in Table 1. Estimated power of constant gravitational waves is enough for rocking celestial objects, which gives reason to hope for their visualization through giant telescopes. Probably traveling gravitational waves are responsible for these rocking, while standing forms of these waves are responsible for the localization of celestial bodies in space. The estimated range of the frequencies is within the sensitivity of Laser Interferometer Space Antenna (LISA) for detecting gravitational waves, which is planned by ESA-NASA space mission (LISA, 2009). Its working principle is similar to LIGO, but it is considerably larger in scale and sensitive to wavelengths several million times longer. This mission gives hope that very soon a spectrum of gravitational waves emitted by the solar system will be found, which would become a model for the search of habitable exoplanets.

\section{CONCLUSION}

The author's proposals are based on the objective reality of the existence of a material physical vacuum and gravitational waves. This also refers to the two strong splashes in gravitational waves that LIGO observed. This paper is an attempt to expand the idea of probable dynamic structures and the properties of the medium of physical vacuum. The Sun and planets oscillate relatively to their barycenter, and as a system consisting of a set of objects undergo asymmetric fluctuations, which can be the source of gravitational waves. On the basis of the estimation of the probable power of the gravitational radiation from the sun, the planets, the moon and even an 
electron, an equation was derived to calculate the frequencies of their oscillation. As a result, the frequency spectrum of the alleged radiation by the solar system and the value of the frequency of the gravitational wave background were obtained. The frequency of the background gravitational waves is equal to the square root of the product of the gravitational constant, and postulates the density of physical vacuum; this frequency is equal to about $.06 \mathrm{~Hz}$. For each of the planets, in association with the Sun, its own frequencies of gravitational waves are found. The greatest frequency belongs to sun couple with Mercury, which with the distance of the planets from the sun sequentially decreases. Accordingly, the wavelength $(\lambda=c / \omega)$ increases; in doing so, $\lambda$ integer once fits inside the length of the orbit of the planet, which the property of the standing wave. The work performed by the waves of physical vacuum is equal to the kinetic energy of the orbital motion of the planets and points to the likelihood of

\section{REFERENCES}

Abbott, B., Abbott, R., Abbott, T., Abernathy, M., Acernese, F., Ackley, K., Adhikari, R. (2016). GW151226: Observation of gravitational waves from a 22-solar-mass binary black hole coalescence. Physical Review Letters, 116(24), 241103. web: http://journals.aps.org/prl/pdf/10.1103/PhysRevLett.116.24 $\underline{1103}$

Crutcher, R. (1982). The local interstellar medium. The Astrophysical Journal, 254, 82-87.

Grimes, R. (1976). Radii for F, Atomistic Simulation Group,. Department of Materials at Imperial College London, http://abulafia.mt.ic.ac.uk/shannon/radius.php?Element=F.

Herbers, T., Elgar, S., Guza, R., \& O'Reilly, W. (1995). Infragravity-frequency $(0.005-0.05 \mathrm{~Hz})$ motions on the shelf. Part II: Free waves. Journal of Physical Oceanography, 25(6), 1063-1079. doi: http://www.whoi.edu/science/AOPE/dept/Publications/044. pdf their participation as the source of this energy. For the detection of the supposed gravitational wave frequencies, an interferometer similar to the LIGO interferometer that is only sensitive to wavelengths several million times longer is needed. Designed by ESA-NASA, the Laser Interferometer Space Antenna (LISA) would be able to offer the measurements of such a range of wavelengths. Constant gravitational waves are reflected in the behavior of celestial bodies and can be visualized by the means of telescopic observation. The possible spectrum of the gravitational waves of the solar system could become a model for the identification of habitable exoplanets. Taken together, the results of this study are limited to show the properties of the host medium and decisive role of cosmic (physical) vacuum in the dynamics and structure of various systems of observed objects.

LISA. (2009). Laser Interferometer Space Antenna (LISA). 12-13,

https://lisa.gsfc.nasa.gov/Documentation/Astro2010_RFI201 2_LISA.pdf.

Noyes, D. (2014). New ATLAS results at LHCP: Higgs mass to string balls. from CERN Website, http://cds.cern.ch/record/1998770

Rosetta blog. (2014). The singing comet, European Space Agency (ESA). posted 2014 by Claudia, http://blogs.esa.int/rosetta/2014/11/11/the-singing-comet/.

Wilson, C., Johansson, G., Pourkabirian, A., Simoen, M., Johansson, J., Duty, T., . . . Delsing, P. (2011). Observation of the dynamical Casimir effect in a superconducting circuit. Nature, 479(7373), 376-379.

Zhussupov, M. (2015). The physical applicability conditions of the Bjerknes equation in dynamics of planetary systems. IAU General Assembly, 22, 45572.

http://adsabs.harvard.edu/abs/2015IAUGA..2245572Z 